\title{
¿LABRA FICTILIA? UNA PROPUESTA INTERPRETATIVA PARA TRES TERRACOTAS DE- coradas procedentes de Segisamo (Sasamón, Burgos) y Villabermudo (PALENCIA)
}

\section{Labra fictilia? An interpretative proposal for three decorated terracotta fragments from Segisamo (Sasamón, Burgos) and Villabermudo (Palencia)}

\section{JESÚS GARCÍA SÁNCHEZ ํㅡ, JOSÉ MANUEL COSTA-GARCÍA²}

(1) Instituto de Arqueología de Mérida. CSIC - Junta de Extremadura. j.garcia@iam.cisc.es

(2) Universidade de Santiago de Compostela. Universidad de Salamanca.jmcostagarcia@usal.es

\section{RESUMEN:}

En este trabajo analizamos tres piezas realizadas en terracota: las dos primeras son originarias del yacimiento de Tisosa (Sasamón, Burgos), mientras que la restante proviene de la villa de Villabermudo (Palencia). Su morfología y semejanzas decorativas nos permiten plantear una nueva interpretación de las mismas como posibles labra fictilia -también denominados louteria o alvei-, esto es, recipientes contenedores de agua para su uso en ambientes domésticos. Este estudio se completa con los resultados de varios análisis arqueométricos cuyo fin ha sido estudiar las pastas y el proceso de fabricación de las piezas halladas en Tisosa.

Palabras clave: arqueometría, terracota, labra fictilia, Segisamo, arqueología romana.

\begin{abstract}
:
We present the study of three Roman terracotta fragments. Two of them were located at the site of Tisosa (Sasamón, Burgos), the third artefact was retrieved during the excavations of the villa at Villabermudo (Palencia). The morphological and decorative similarities brought us to present an interpretive hypothesis as labra fictilia, also called louteria or alveus to define water containers for domestic use. This study is complemented with archaeometry research, which provided insightful details of fabric and the manufacturing process of the artefacts located at Tisosa.
\end{abstract}

Key words: archaeometry, terracotta, labra fictilia, Segisamo, Roman archaeology. 
Desde 2009, el territorio de la ciudad romana de Segisamo -bajo el solar de la actual Sasamón, Burgos- ha sido objeto de estudio en el marco de un programa de investigación cuya finalidad es conocer mejor el modo en que se articularon los paisajes arqueológicos entre la Segunda Edad de Hierro y la Antigüedad Tardía en el valle del río Duero (fig. 1). En sus primeras fases, la investigación se centró en el estudio espacial del poblamiento desde una perspectiva cronológica amplia (García Sánchez 2009; 2012), re-orientándose más recientemente al mapeado y catalogación detallada de evidencias arqueológicas en esta zona, así como a la prospección y excavación del oppidum prerromano del Cerro de Castarreño (Olmillos de Sasamón, Burgos) (García Sánchez y Costa-García 2019). Este enclave se ha relacionado recurrentemente con los Turmogi o turmogos (en ocasiones también denominados turmódigos), un pueblo indígena situado en la periferia del mundo vacceo, ubicado a su vez en torno al cauce medio del río Duero (Teja Casuso 1991; Solana Sainz 2001; Francés Negro 2011; García Sánchez 2019) y al S del territorio de los Cantabri, cuyos grandes oppida como La Ulaña, Monte Bernorio, Icedo o Coculina pueden verse desde la vega de los ríos Pisuerga, Odra y Brullés.

Así, pretendemos ahondar en el conocimiento de las profundas transformaciones vividas en el territorio con motivo de la expansión del estado romano, proceso durante el cual las comunidades indígenas locales sufrieron un cambio de paradigma cultural equiparable al de muchas otras poblaciones que habitaron la Meseta Norte. Tras la conquista romana -proceso definitivamente concluido después de las campañas de 29-19 a.C. contra Cantabri y Astures- se produjo el abandono de los grandes oppida que caracterizaban a la organización sociopolítica precedente (Sacristán de Lama 2011). Este proceso ha de relacionarse con la fundación ex novo de ciudades romanas, llamadas a ser los centros administrativos de la región (López Noriega 1997; 1998; Martínez Caballero et al. 2018).

En este contexto, la ciudad de Segisamo surge en un momento indeterminado del último cuarto del s. I a.C. (Abásolo y García 1993), muy posiblemente en relación con la reestructuración experimentada en la región a consecuencia de las campañas augusteas contra los Cantabri. Aunque las fases de ocupación más antiguas de la ciudad son mal conocidas arqueológicamente, sabemos que el asentamiento de la legio IIII Macedonica en la vecina Herrera de Pisuerga -antigua Pisoraca- tuvo un enorme impacto en la cuenca del río Pisuerga y sus afluentes (Morillo Cerdán 2000; Gómez Pantoja 2000).
El hallazgo de numerosos cipos o termini delimitando los prata legionis del territorio de las ciudades de Iuliobriga -ager Iuliobrigensis- al N y de Segisamo -ager Segisamonensis- al S, así parecen indicarlo (Cortés Bárcena 2009). De la implicación de esta unidad militar en la ordenación del territorio hispano a través de la construcción de infraestructuras de comunicaciones tenemos testimonios en los miliarios de la vía de las Cinco Villas (Zaragoza) (Lostal Pros 2009) o en los sillares del Pont del Diable (Martorell, Barcelona) (Gurt y Rodá de Llanza, 2005; Rodá de Llanza 2006). En relación con el ambicioso plan urbanístico iniciado en la meseta $\mathrm{N}$ durante la posguerra, sabemos que la legión jugó un papel activo en la monumentalización de la capital conventual, Clunia Sulpicia (Coruña del Conde, Burgos) (Palao Vicente 2017: 59; Morillo Cerdán y Salido Domínguez 2013: 311-12).

Durante las prospecciones de 2017 recuperamos además en Segisamo-Sasamón un sello con numerales sobre un fragmento de material constructivo (García Sánchez et al. 2020). Esta pieza, todavía inédita, viene a sumarse a otras evidencias materiales y estructurales relacionables con la presencia militar romana en la zona, todavía en fase de estudio (Didierjean et al. 2014; García Sánchez y Costa-García 2020).

En los últimos años hemos basado nuestra estrategia de investigación en la prospección arqueológica para la detección de nuevos yacimientos (Menéndez Blanco et al.2020) y para documentar espacios de actividad pretéritos, tanto de ocupación como de actividades económicas relacionadas con el medio rural. Esta dialéctica entre el registro arqueológico convencional a escala de sitio o yacimiento y de no-sitio (off-site) ha sido conceptualizada en numerosas experiencias de trabajo en el Mediterráneo Oriental y Central, dando lugar a un interesante debate sobre métodos e interpretaciones del registro arqueológico de superficie (Cherry et al. 1991; Fentress 2000; Blanton 2001). También ha sido empleado en la arqueología peninsular en diversos casos de estudio paradigmáticos como La Serena (Badajoz) (Mayoral Herrera y Uriarte 2011; Sevillano Perea et al. 2013) o en la serranía alicantina (Grau Mira 2017).

Durante el transcurso de las prospecciones orientadas al registro off-site en el territorio de Sasamón (García Sánchez y Cisneros Cunchillos 2013) localizamos en 2010 el yacimiento inédito de Tisosa (García Sánchez y Cisneros 2014). Situado en la orilla derecha del río Brullés y próximo a la ciudad de Segisamo, este yacimiento fue 


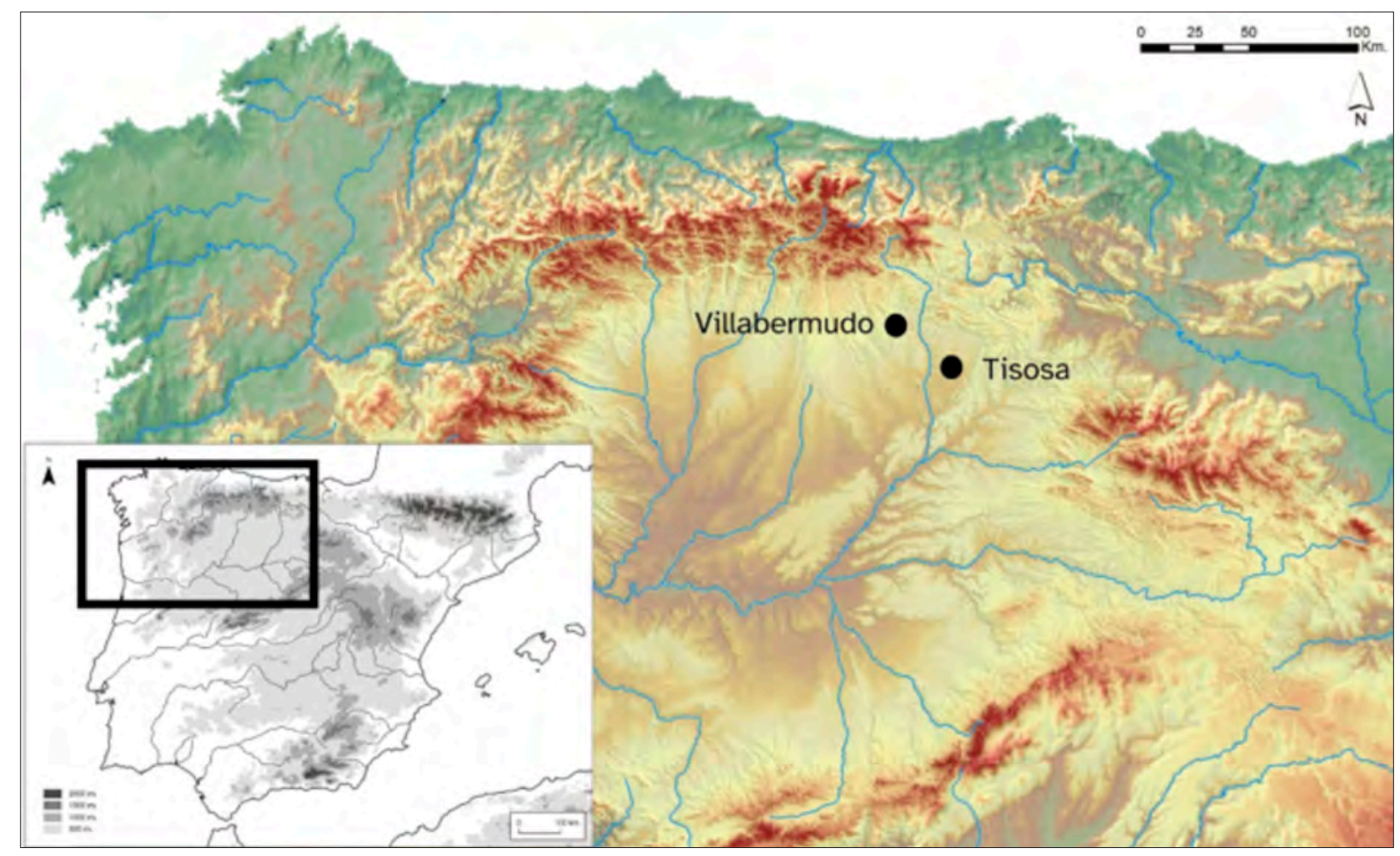

Fig. 1: Ubicación de los yacimientos de Tisosa (Sasamón) y Villabermudo en el contexto de la meseta Norte (Autor: Jesús García Sánchez).

interpretado en primera instancia como un posible establecimiento suburbano subordinado a la ciudad romana. Los materiales arqueológicos allí hallados constituyen la espina dorsal de este trabajo.

\section{PROCEDENCIA DE LAS PIEZAS}

\section{EL YACIMIENTO DE TISOSA}

Durante la prospección arqueológica desarrollada en 2010 se localizó una concentración de material muy densa que incluía abundante material constructivo romano, cerámica común, terra sigillata hispánica, fragmentos de mosaicos y algunos fragmentos de ánfora (García Sánchez y Cisneros Cunchillos 2014, 131-133). La tipología de los fragmentos anfóricos han sido identificados con las formas Dressel 1 de origen campano -un pivote-, Haltern 70 del valle del Guadalquivir y tipos rodios (García Sánchez y Cisneros 2014; Carreras y Morais 2004). Esto tipos se datan en los momentos finales del s. I a.C., proporcionando una datación post quem que se sitúa en el mismo horizonte cronológico en el que se efectúa la fundación de la ciudad romana. El resto de materiales cerámicos, TSH y TSHt, evidencian la perduración del yacimiento en el tiempo, hasta al menos el s. III d.C.Aunque inicialmente confundida con un fragmento de tégula romana, se localizó durante estas prospecciones la primera terracota decorada que analizamos en este trabajo (número de inventario 10.21.100/ museo de Burgos MBU-9961) (fig. 2).

Por el momento desconocemos la funcionalidad exacta del yacimiento, ya que no se han realizado excavaciones o prospecciones geofísicas en la zona. $\mathrm{Su}$ interpretación funcional se basa en el análisis de fotografías aéreas realizadas en 2010 y 2020 , así como en los resultados de las mencionadas prospecciones artefactuales. De la información arqueológica acumulada se colige que estamos ante un complejo edilicio de al menos 1,5 has de extensión, quizás compuesto por diferentes estructuras con suelos decorados con mosaicos a bandas tricolores como los localizados en superficie y habitado posiblemente por individuos de origen o gustos itálicos. Esta hipótesis no solo se fundamenta en las mencionadas importaciones egeas y campanas, sino también en el borde de un plato de engobe rojo interno "pompeyano", indicativo de una cocina al estilo romano (Girón y 


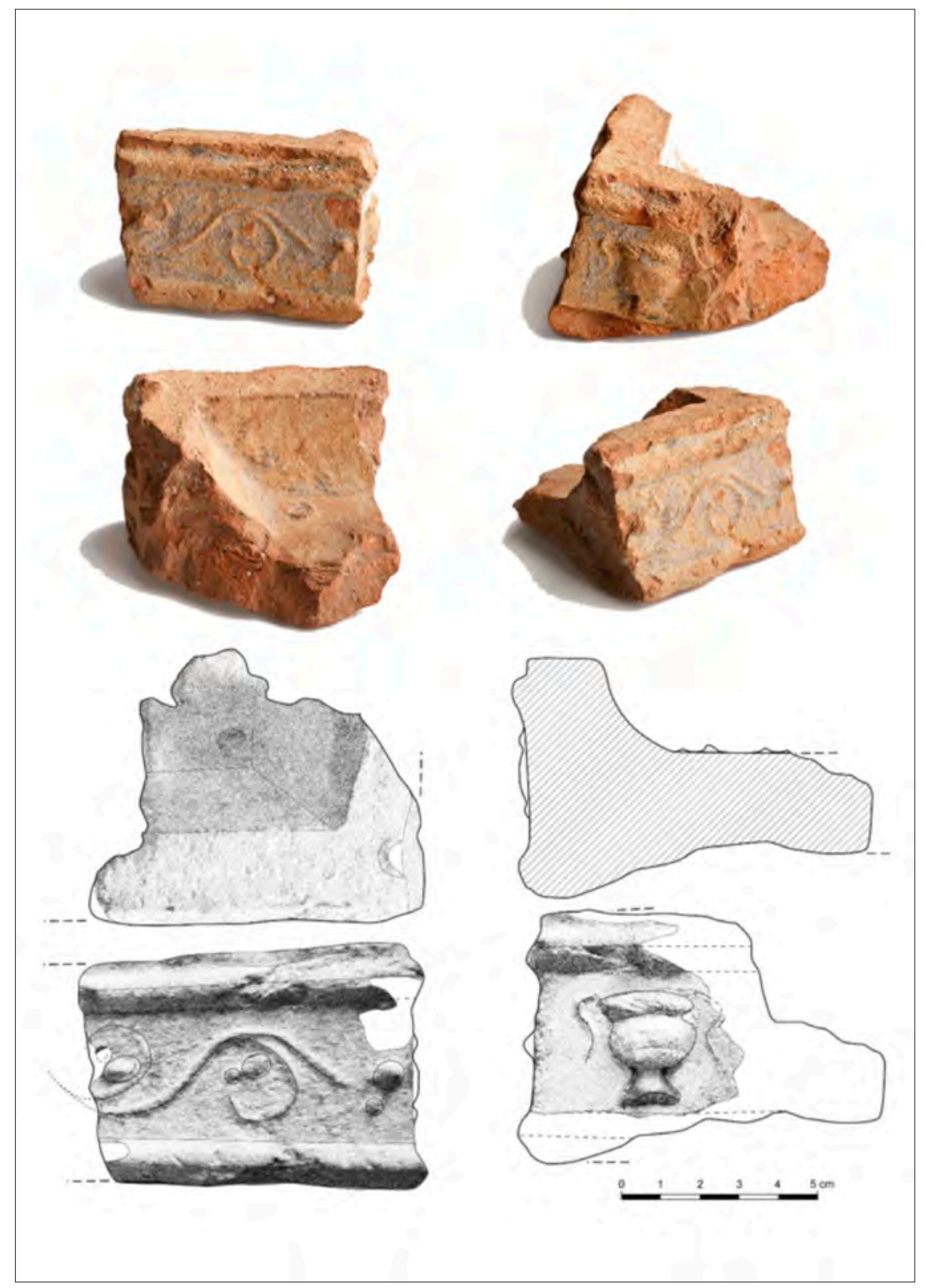

Fig. 2: Fotografía y dibujo de la pieza 1, MBU-9961. Fotografía de Julián Romeo y dibujo de Maricruz Sopeña, montaje: Jesús García Sánchez.

Costa-Garcia 2009). Cabe señalar, además, que la producción de las Haltern 70 del valle del Guadalquivir se ha relacionado con la esfera militar (Carreras y Morais 2004; Lapuente et al. 1996).

Por lo que respecta a la segunda pieza ( $\mathrm{n}^{\circ}$ de inventario 17.17.2005) (fig. 3), fue entregada por un vecino de Sasamón a los autores de este trabajo durante la campaña de prospección Warscapes: documentando la presencia militar romana en la campiña ( $\mathrm{N}^{\mathrm{o}}$ de expediente 31-17BU) (García Sánchez et al. 2020) ${ }^{1}$. De acuerdo con sus indicaciones, procede de la misma finca prospectada por nuestro equipo en 2010 y conocida como Tisosa. Con las debidas precauciones, hemos considerado que su contexto es, por tanto, idéntico al de la primera pieza descrita.

\section{LA VILLA DE VILLABERMUDO}

El tercer fragmento analizado en este trabajo procede del yacimiento romano de Villabermudo (Herrera de Pisuerga, Palencia) y se encuentra actualmente en 
Fig. 3: Fotografía y dibujo de la pieza 2, 17.17.2005. Fotografía de J. García Sánchez y dibujo de Lisa Götz.

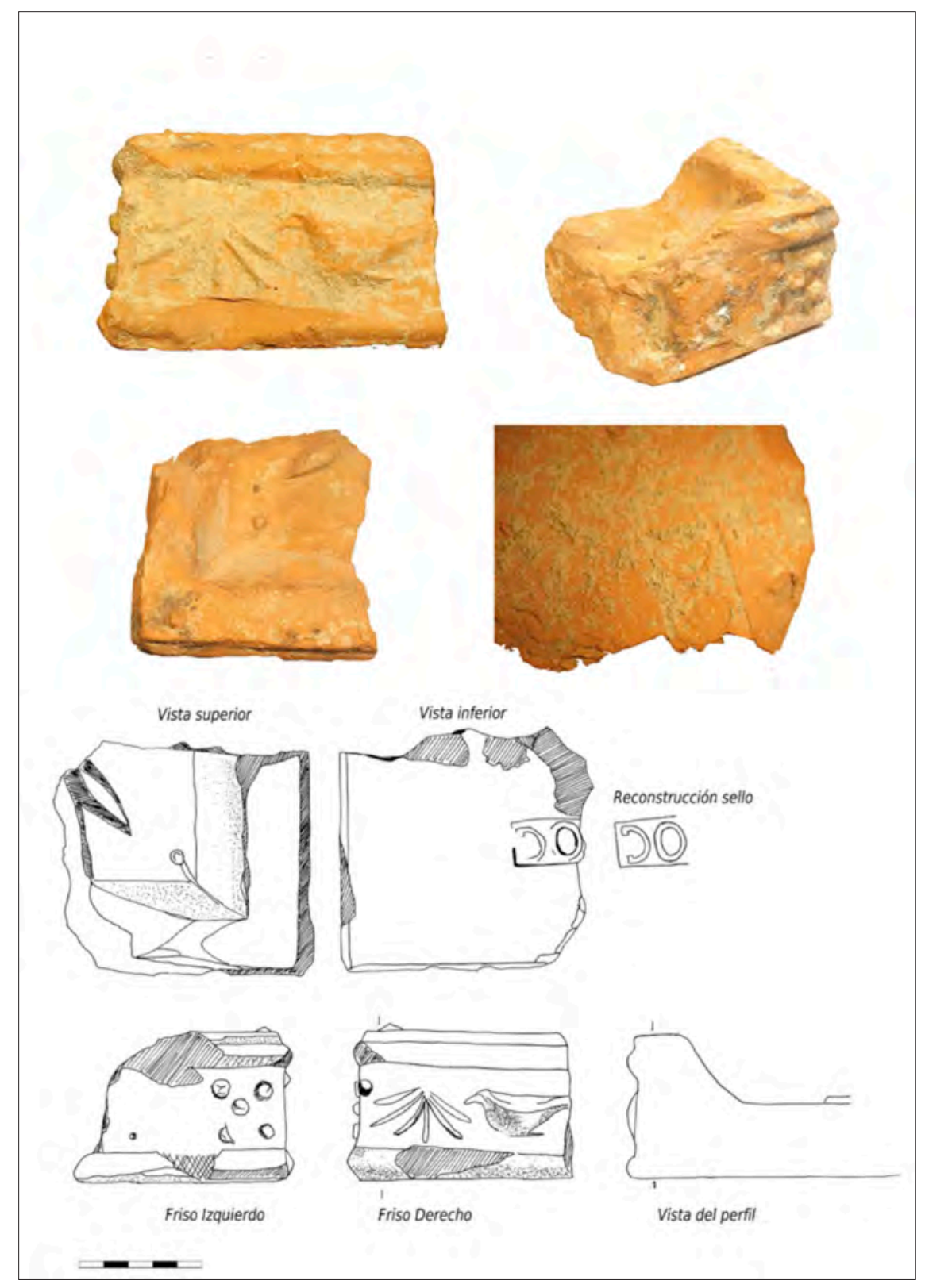

prácticamente la totalidad de dichas cajitas (Pérez González y Illarregui 1990) (fig. 4).

Recientemente, Pérez González ha reseñado las intervenciones en la villa romana, atribuyendo la totalidad del yacimiento al periodo romano y clasificando estas piezas bajo el tipo "cajitas romanas" (Pérez González 2017: 125). Para el fragmento que nos ocupa propone una cronología amplia -ss. I-III d.C.-, aunque la encuadra preferentemente en el s. II d.C. por haberse hallado en los niveles más antiguos de la villa (Pérez González 1993: 14). zada a molde y no a navaja, como suele suceder en 


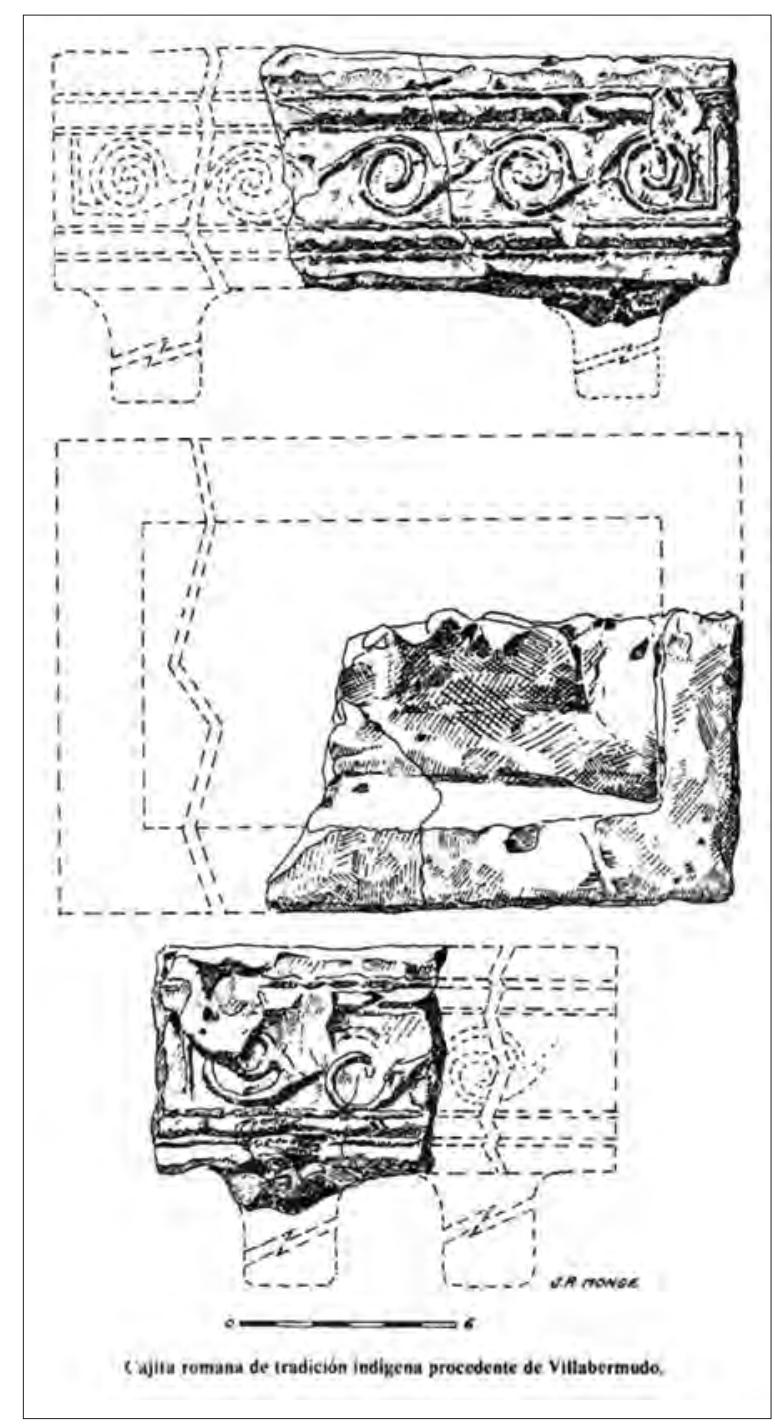

Fig. 4: Dibujo de la pieza de Villabermudo. Autor Cesáreo González.

\section{DESCRIPCIÓN FORMAL Y DECORACIÓN DE LAS PIEZAS}

\section{PIEZA 1. MBU-9961}

La primera pieza proveniente de Tisosa mide $6 \mathrm{~cm}$ de alto por $8,4 \mathrm{~cm}$ de largo en su parte mejor conservada. El exterior está formado por un friso de $3,4 \mathrm{~cm}$ de ancho y 8,1 $\mathrm{cm}$ de largo en el lado mejor conservado y de $5,1 \mathrm{~cm}$ de largo en el lateral contiguo. Los frisos están enmarcados por una moldura superior e inferior de $1,1 \mathrm{~cm}$. Desde una perspectiva cenital los bordes del friso poseen una anchura de 2,2 cm. El borde interior presenta una pared curva que finaliza en la parte interna de la pieza, parece estar cubierta por una pátina fina muy adherente. La parte inferior está rota, aunque hay restos de mortero adherido (fig. 2).

La decoración del primer friso está compuesta por una guirnalda de roleos en espiral, que finalizan en un aplique o botón en relieve. El friso contiguo presenta una vasija cerámica globular con pie alto, quizás una crátera flanqueada por dos espirales. La decoración de ambos frisos se realizó a molde. El color de esta pieza es naranja rojizo 7.5YR 7/6.

\section{PIEZA 2. 17.17.2005}

La segunda pieza tiene una altura de $6,1 \mathrm{~cm}$ de altura por $8,4 \mathrm{~cm}$ en su extremo mejor conservado. El friso mide $3,3 \mathrm{~cm}$ y las molduras que lo enmarcan miden 1,2 $\mathrm{cm}$. El ancho de los laterales es de 2,1 cm. Como vemos, las medidas son prácticamente iguales a las de la pieza 1 . Su parte interior tiene también una pared curva en cuya diagonal se dispone la decoración de palmeta con perspectiva cenital (fig. 3). Esta última decoración parece haber sido realizada a posteriori, aplicándose a barbotina al interior de la pieza.

En el primer friso de la pieza encontramos cinco perlas o apliques plásticos dispuestos de forma que recuerdan a la cara de un dado: dos en la parte superior, uno en el centro $\mathrm{y}$ dos en la parte inferior. Estos apliques se realizaron muy probablemente con la técnica de la barbotina El friso contiguo es quizás más expresivo, pues representa una hoja invertida con cinco nervios y un pájaro con las alas plegadas orientado hacia dicha hoja. El color de la pasta es de color anaranjado de tonalidad amarillo 5YR 7/8.

Este fragmento presenta elementos que no aparecían en la primera pieza. El primero de ellos es un sello en la parte inferior que no conserva completo, pero en el que puede reconocerse la leyenda $D O[. .$.$] . Este sello podría$ reflejar un nombre común domo Domitius, o bien referirse a la fórmula (Opera)Do(liare), una forma habitual de referirse a piezas constructivas como ladrillos, tejas, tuberías, piezas decorativas (Berni Millet 2008: 130). Sabemos, además, que esta parte inferior no era visible, pues en la primera pieza se detectó presencia de mortero para poder fijarse a un soporte. El segundo elemento a destacar -más relevante si cabe-, es la presencia de una hojita -la parte conservada no permite esclarecer si es una hoja de laurel, lanceolada o de agua- y un pequeño botón en relieve en la parte interior de la pieza. Esta 
decoración nos hace pensar que esta parte sí era visible, por lo que debemos descartar que sobre este friso se localizase otra estructura, tal vez un tejado compuesto por tégulas, ímbrices y antefijas en miniatura.

\section{PIEZA 3}

Sus dimensiones divergen solo ligeramente de aquellas registradas en las dos piezas halladas en Tisosa, pues mide $13 \mathrm{~cm}$ en el lado mayor, $8 \mathrm{~cm}$ en el menor y alcanza los $7 \mathrm{~cm}$ de altura. El friso mide $2,5 \mathrm{~cm}$ y está enmarcado por las consabidas molduras de aproximadamente $1 \mathrm{~cm}$ de anchura. El ancho de los laterales alcanza los 2,1 cm. Por su parte la decoración está compuesta en ambos lados por roleos en espiral entrelazados. Según el dibujo proporcionado por sus investigadores (Pérez González 1993: 20), podría conservar el arranque de una pata, quizá sustento para su interpretación como cajita. Este elemento solo se ha documentado en esta pieza, pero no nos ha sido posible reconocerlo en persona al no conocerse el paradero de este objeto (fig. 4).

\section{ANÁLISIS ARQUEOMÉTRICOS}

Una primera observación macroscópica de las piezas 1 y 2 -las únicas localizables- parecía indicar que, a pesar de la similitud formal, nos encontrábamos antes dos objetos diferentes. Habida cuenta de la indefinición tipológica a la que nos enfrentábamos, planteamos un análisis arqueométrico detallado de estas piezas con el objeto de contextualizar mejor su composición y obtener nueva información sobre su proceso de manufactura.

Así pues, se llevaron a cabo dos análisis con estas piezas: una difracción de rayos $\mathrm{X}$ y una lámina delgada ${ }^{3}$. Ambas técnicas son de sobra conocidas en el estudio petrográfico de la cerámica en contextos arqueológicos (Quinn 2013), por lo que no describiremos detalladamente los procedimientos seguidos. No obstante, queremos reseñar que nuestro estudio hace especial hincapié en las modificaciones de la composición mineralógica como clave para comprender el proceso tecnológico de fabricación de las piezas (Linares et al. 1983: 482). En primer lugar, la difracción de rayos $\mathrm{X}$ busca conocer los tipos de materiales, su cantidad y los posibles procesos técnicos (temperatura de cocción) que han tenido como resultado la composición y factura actual de las pastas. El segundo método, empleado para su estudio comparativo actual y futuro, consiste en la realización de láminas delgadas de ambas piezas y su fotografiado en un microscopio petrográfico para su posterior estudio mineralógico.

La difracción de rayos X (fig. 5) practicada en la pieza 1 revela la existencia de gehlenita, un material de neoformación que aparece al alcanzar temperaturas de entre $800^{\circ} \mathrm{C}$ y $950^{\circ} \mathrm{C}$ para posteriormente descomponerse en $\mathrm{CaO}$-óxido de calcio-. La ausencia de una fase de neoformación de diópsido indicaría que la temperatura de cocción alcanzada fue de entre 950 y $1000^{\circ} \mathrm{C}$.

Como se aprecia en los gráficos de difracción y en las estadísticas para la pieza 2 , la diferente proporción de moscovita y cuarzo indica diferencias en la materia prima utilizada, ya observables en el examen macroscópico. Además, esta pieza sí muestra la mencionada fase de neoformación del diópsido y la descomposición de la gehlenita, además de un mayor contenido en illita. Estos datos nos sirven como geotermómetros, permitiéndonos apuntar que la temperatura de cocción es ligeramente inferior a la primera muestra, es decir de entre $900^{\circ} \mathrm{C}$ y $950^{\circ} \mathrm{C}$.

En cuanto al estudio de las láminas delgadas (fig. 6), la pieza 1 presenta clastos de carbonatos muy abundantes, cuarzo, cuarcita y cemento ferruginoso. La pieza 2 presenta cemento ferruginoso con abundantes clastos de cuarzo, cuarcita, carbonatos, micas accesorias y fragmentos de rocas carbonatadas. La figura 7 muestra los porcentajes para ambas muestras.

Como ya se avanzaba anteriormente, las piezas están en efecto compuestas por materias primas diferentes, pues así se deduce de la proporción entre moscovita y cuarzo. Los valores similares de diópsido y gehlenita indican una leve diferencia en la temperatura de cocción que sin embargo no sería extraña en procesos productivos serializados. De este modo, se concluye que los materiales empleados son realmente similares y las diferencias que encontramos en la tabla de semicuantificación se deben principalmente a la neoformación de materiales en función de la temperatura alcanzada en el horno. Podemos especular con una producción en un mismo taller con ligeras variaciones y con modelos diferentes, siempre realizados con la técnica del molde.

\section{¿POSIBLE LABRA FICTILIA?}

En 2014 -cuando se dio a conocer el yacimiento de Tisosa- ofrecimos una primera aproximación cronológica y funcional basándonos únicamente en la pieza 1 , junto 


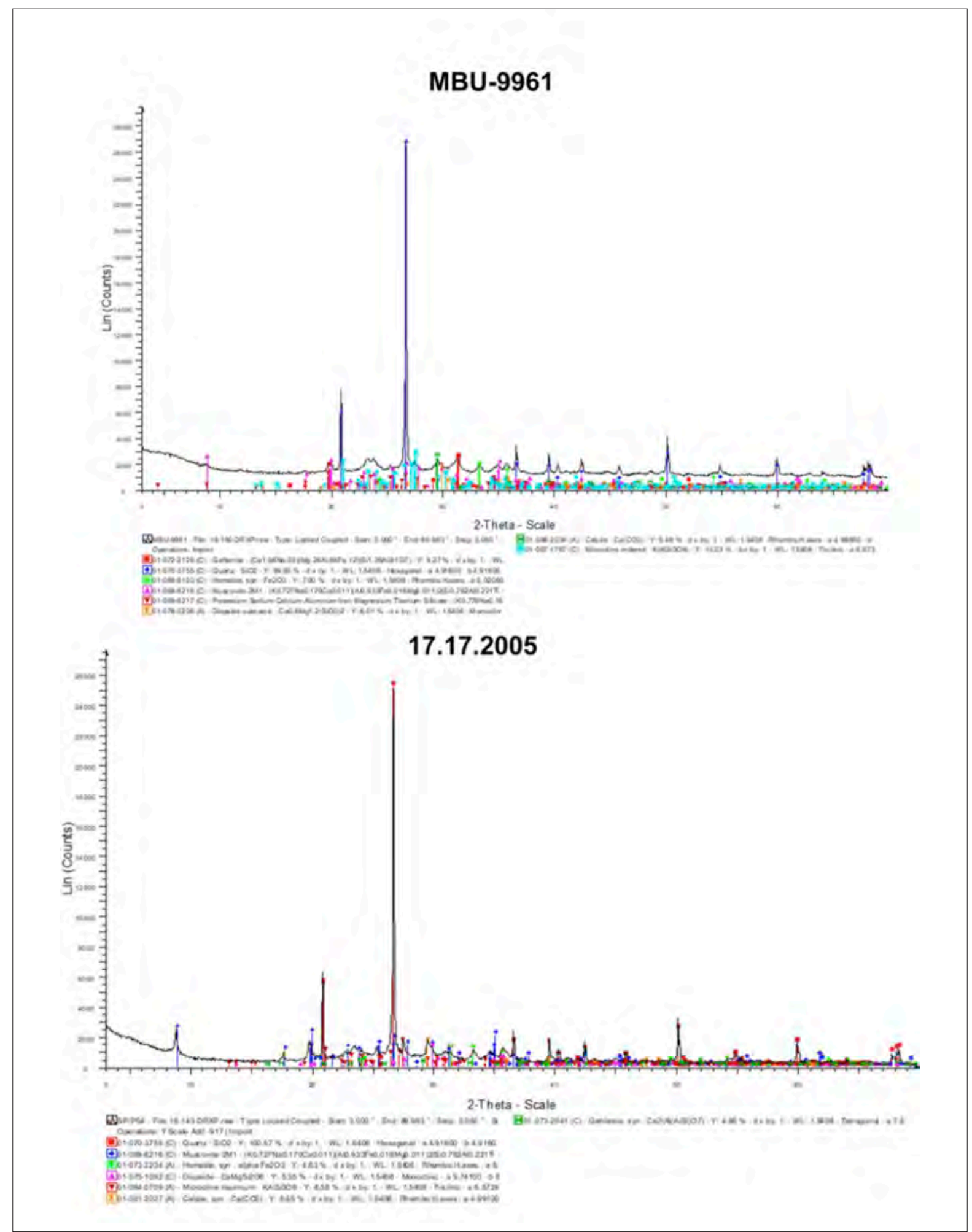

Fig. 5: Gráficos de Difracción de Rayos X para las piezas 1 y 2 Autor: María Candelas Moro, Servicio de Difracción de Rayos X de la USAL, montaje: Jesús García Sánchez. 


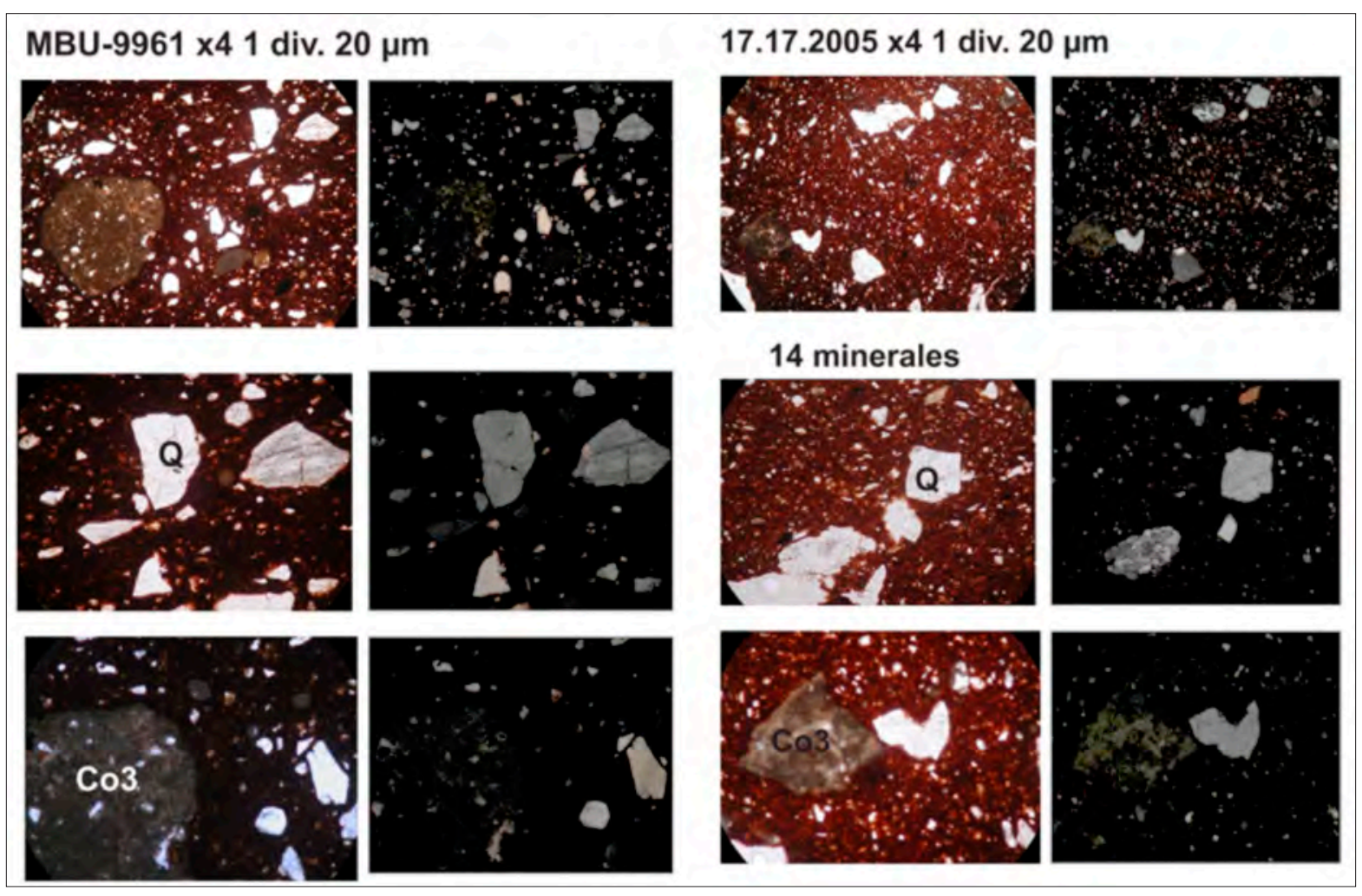

Fig. 6: Lámina delgada de piezas 1 y 2. Autor: María Candelas Moro, montaje: Jesús García Sánchez.

con el conjunto material localizado en la prospección superficial (García Sánchez y Cisneros Cunchillos 2014). La ausencia de paralelos nos llevó a interpretar el fragmento como parte de un pequeño altar o larario, del que la pieza parte de un friso. Esta hipótesis se basaba en las pequeñas dimensiones del fragmento y la existencia bien conocida de antefijas de pequeño tamaño (Ramos Sainz 1996: 201-202), seguramente también parte de la decoración de un edificio en miniatura.

La aparición de la pieza 2 en 2017 aporta nuevos indicios para comprender la forma y función del objeto. Como hemos señalado, la decoración interior cuenta con una hoja de laurel, lanceolada o de agua. La parte inferior a pesar de estar sellada podría no ser visible, ya que existen indicios que nos hacen sospechar que estuviese sujeta a otro elemento. Si la ubicación del sello $D O$ es representativa del centro de la pieza, nos encontraríamos ante un objeto de entre 15 y $20 \mathrm{~cm}$ de lado. El bisel que forman las paredes del friso hacia el interior de la pieza, junto a la decoración de la hoja en perspectiva cenital son quizá indicativos de que la parte interior sirviese para contener un líquido o un sólido.

\begin{tabular}{|l|c|c|}
\hline & Pieza 1 (MBU-9961) & Pieza 2 (17.17.2005) \\
\hline Cuarzo & 40 & 32 \\
\hline Moscovita & 22 & 32 \\
\hline Hematita & 3 & 3 \\
\hline Diópsido & 10 & 9 \\
\hline Microclina & 17 & 18 \\
\hline Calcita & 3 & 3 \\
\hline Gehlenita & 5 & 3 \\
\hline SUMA & 100 & 100 \\
\hline
\end{tabular}

Fig. 7: Semicuantificación de los materiales en lámina delgada para las dos piezas procedentes de Tisosa (Sasamón).

Una posibilidad sugerente es que se trate de labra fictilia o alvei. Las fuentes ornamentales han sido estudiadas por Morillo y Salido (2011: 155; 2010: 168) para la Península Ibérica, representándose además en los frescos de la villa A de Popea en Oplontis un ejemplar muy similar a las piezas que nos ocupan (Morillo y Salido 2011: 156, fig. 4). Con todo, los alvei conservados son elementos 


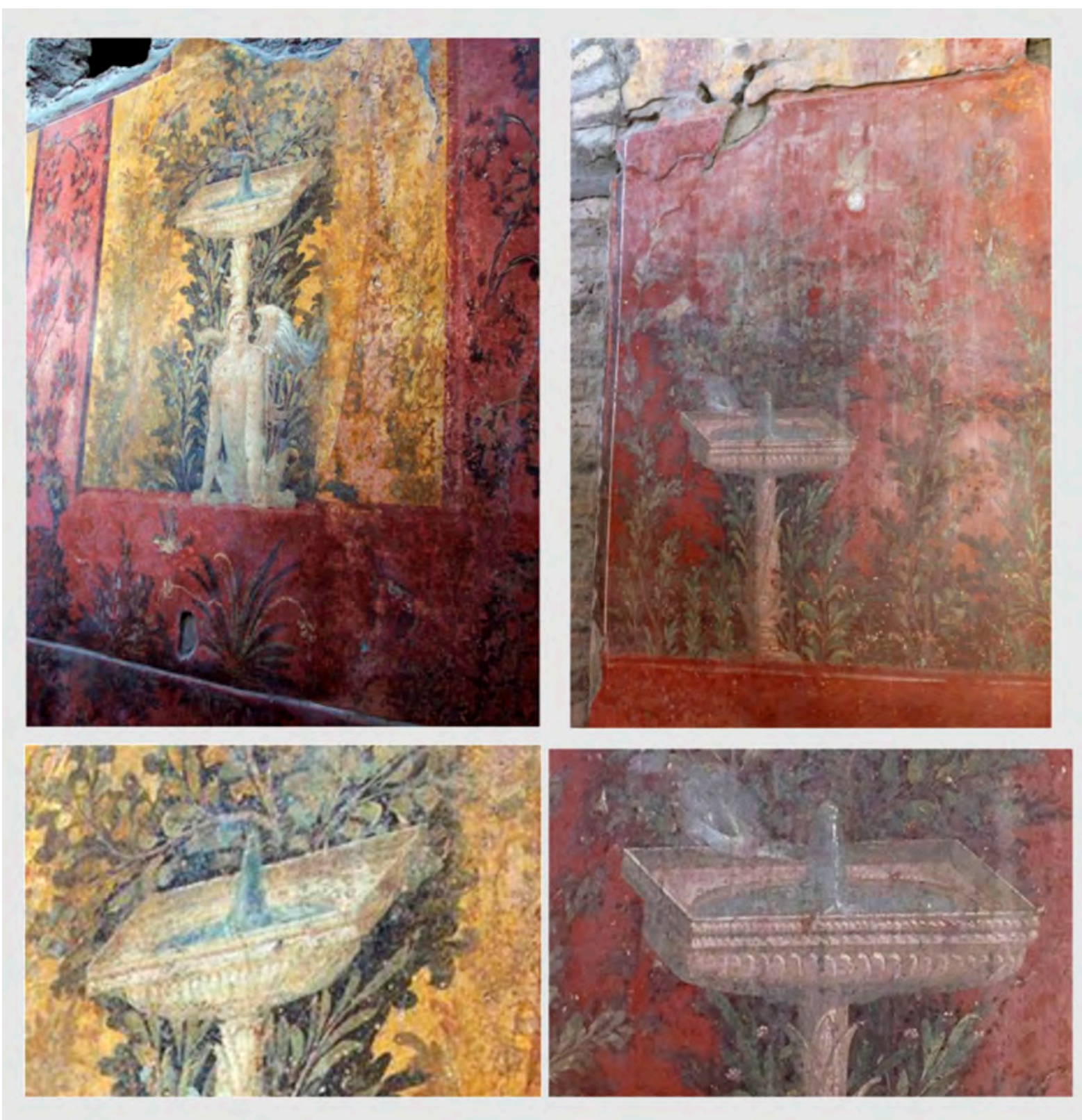

Fig. 8: Representación de louteria o alveus decorativos en frescos de la villa de Popea en Oplontis, Campania. Fuente: http://incampania. com/en/location/archaeological-excavations-of-oplontis-en/ Creative Commons. Montaje: Jesús García Sánchez.

fabricados en mármol y no contamos con paralelos conocidos fabricados en material cerámico. Más allá de su uso como tales fuentes, se recoge además de forma somera en Columela la existencia de lebrillos de barro o piedra utilizados para secar higos -labra fictilia ${ }^{4}$ o bien contenedores para recoger el aceite recién exprimido -fictilia labra ${ }^{5}$ - (fig. 8).
Huguet (2013: 315) confirma que los louteria de origen griego, realizados en pastas locales, se caracterizan por su gran diámetro, base plana, paredes bajas y están decorados tanto en el borde como el interior, con motivos geométricos o vegetales. De nuevo en Pompeya encontramos referencias a estos elementos de origen griego (Fergola y Scatozza-Höricht 2001). Estos louteria se 
destinan a contener agua en ambientes públicos donde se realizarían ejercicios - palestrae- y precederían a la popularización de los labra domésticos confeccionados en mármol (Fergola y Scatozza-Höricht 2001: 165).

A partir de esta documentación podemos deducir que las piezas que nos ocupan posiblemente se corresponden con recipientes destinados a contener líquidos, pudiendo usarse eventualmente como para contener agua a modo de alveus. La decoración de las piezas aquí presentadas nos permite descartar su uso meramente productivo.

\section{CONCLUSIÓN}

En este breve trabajo hemos contextualizado las piezas 1 y 2 como elementos procedentes del yacimiento de Tisosa, un complejo construido en torno al cambio de era en el área periurbana de Segisamo (Sasamón). En este lugar encontramos elementos que nos informan del gusto o capacidad de los habitantes de este lugar para obtener productos importados del centro y el oriente del Mediterráneo, transportándose en algunos casos en los ya mencionados contenedores anfóricos. En este horizonte de consumo debemos ubicar las terracotas decoradas que proponemos puedan clasificarse como en alvei o incluso labra fictilia.

Con todo, la ausencia de paralelos conocidos para estas piezas, así como lo reducido de la muestra analizada, nos impiden definir su origen y funcionalidad exactos. Únicamente podemos conjeturar que en época temprana un alfarero o alfareros conocedores de las las decoraciones itálicas y de los modelos griegos/itálicos de alveus o louteria fabricaron estas piezas. El análisis arqueométrico concluye que las piezas 1 y 2 fueron fabricadas separadamente empleando pastas ligeramente diferentes, pero siguiendo un mismo proceso técnico. La decoración se aplicó empleando el molde.

En este contexto resulta inevitable pensar en la proximidad de Herrera de Pisuerga y las particulares características de la producción y consumo cerámicos documentados aquí hacia el cambio de era (Morillo Cerdán 1992; Morillo Cerdán et al. 2006; González y Gómez 2006; Gómez Pantoja 2000). Sin embargo, en el actual estado de conocimiento solo podemos esperar que los datos provistos por las láminas delgadas y la difracción de rayos X puedan ayudar a contextualizar estas y otras piezas en el futuro. Igualmente, la localización y estudio de la extraviada pieza de Villabermudo contribuiría a conocer mejor estos interesantes elementos y a establecer paralelismo con esta área.

\section{NOTAS}

1. Agradecemos a Carlos Gallego la entrega de esta pieza para su estudio y conservación en el Museo de Burgos.

2. Agradecemos a Francisco Javier Abarquero del Museo de Palencia sus intentos de ubicar la pieza, que por el momento se mantiene en paradero desconocido.

3. Los análisis han sido realizados por M. Candelas Moro Benito, profesora titular del Área de Cristalografía Y Mineralogía, Departamento de Geología de la Universidad de Salamanca.

4. Quidam lectis ficis pediculos adimunt et in sole eas expandunt; cum deinde paulum siccatae sunt, antequam indurescant, in labra fictilia vel lapidea congerunt eas, tum pedibus lotis in modum farinae proculcant et admiscent torrefactam sesamam cum aneso Aegyptio et semine faeniculi et cymini (Columella Rust. 12.15.3).

5. Quod deinde primum defluxerit in rotundum labrum - nam id melius est quam plumbeum quadratum vel structile gemellar - protinus capulator depleat et in fictilia labra, huic usui praeparata, defundat (Columella Rust. 12.52.10).

\section{AGRADECIMIENTOS}

Agradecemos a la Dra. M. Candelas Moro Benito (Departamento de Cristalografía y Mineralogía de la Universidad de Salamanca) la realización de los análisis y la lámina delgada de las piezas 1 y 2. A Marta Negro y a Adelaida Rodriguez, respectivamente directora y restauradora del Museo de Burgos, el habernos facilitado el acceso a la pieza 1, ya depositada en sus instalaciones. Finalmente, queremos recordar los esfuerzos del Museo de Palencia por localizar la pieza 3. Agradecemos también a M. de los Reyes de Soto (CSIC) sus comentarios sobre el manuscrito y a Macarena Bustamante (Universidad de Granada) su opinión sobre el sello DO en la pieza 2. Finalmente agradecemos a Julián Romeo y a Maricruz Sopeña las fotos y dibujo de la pieza 1 y a Lisa Götz el dibujo de la pieza 2.

\section{BIBLIOGRAFÍA}

ABÁSOLO, J. A.; GARCÍA, R. (1993): Excavaciones en Sasamón (Burgos), Madrid.

BERNI MILLET, P. 2008- Epigrafía anfórica de la Bética, Barcelona. BLANTON, R. E. (2001): Mediterranean myopia, Antiquity 75, 627-29.

DOI: https://doi.org/10.1017/S0003598X00088918

CARRERAS, C.; MORAIS, R. (2004): Geografia del consum de les Haltern 70m, Culip VIII i les àmfores Haltern 70. Monografies del Casc 5, Barcelona, 93-115.

CHERRY, J. F.; DAVIS, J. L.; MANTZOURANI, E. (1991): Data Evaluation and Off-Site Distribution, Landscape Archaeology as Long-Term History. Northern Keos in the Cycladic 
Islands (J. F. Cherry, D. L. Davis, E. Mantzourani, eds.) Los Angeles, 37-54.

COLUMELlA (1941): On Agriculture, H. B. Ash. Cambridge, Loeb Classical Library. DOI: https://doi.org/10.4159/DLCL.columella-agriculture.1941

CORTÉS BARCENA, C. (2009): El territorio militar en la epigrafía de la Hispania Romana: los Termini Pratorum, Anejo de Gladius 9, 91-101.

DIDIERJEAN, F.; MORILLO CERDÁN, A.; PETIT-AUPERT, C. (2014): Traces de guerres, traces de paix armée: l'apport de quatre campagnes de prospection aérienne dans le nord de l'Espagne, La guerre et ses traces: conflits et sociétés en Hispanie à l'époque de la conquête romaine (IIIe-Ier s. a. C.) (F. Cadiou, M. Navarro, eds.), Bourdeaux, 149-80.

FENTRESS, E. (2000): What are we counting for?, Extracting Meaning from Ploughsoil Assemblages (R. Francovich, H. Patterson, G. Barker), Oxford, 44-52.

FERGOLA, L.; SCATOZZA-HÖRICHT, L. A. (2001): "Louteria” fittili da Pompei, Rivista di Studi Pompeiani 12/13, 143-66.

FERNÁNDEZ VEGA, P. A.; BOLADO DEL CASTILLO, R.; ILLAREGUI, E.; PEÑIL MÍNGUEZ, J. (2010): Hallazgo de dos nuevas cajitas celtibéricas en Camesa-Rebolledo (Valdeolea-Cantabria), Munibe 61, 221-34.

FRANCÉS NEGRO, M. (2011): Los turmogos. Revisión de una etnia poco conocida del Norte, ArqueoUCA 1, 31-40.

GARCÍA SÁNCHEZ, J. (2009): El poblamiento y la explotación del paisaje en la meseta norte entre la edad del hierro y época romana altoimperial. Una aproximación a través de la arqueología espacial, Zephyrus 59 (2), 81-96.

GARCÍA SÁNCHEZ, J. (2012). Arqueología y paisaje en el noroeste de Burgos: la transición de la Segunda Edad de Hierro a época romana a través del registro material, Tesis Doctoral, Universidad de Cantabria, <http://www.tdx.cat/ handle/10803/80486>.

GARCÍA SÁNCHEZ, J. (2019): Los turmogos, sociedad y territorio en los confines del valle del Duero, Vaccea Anuaria, $52-28$.

GARCÍA SÁNCHEZ, J.; CISNEROS CUNCHILLOS, M. (2013): An Off-Site Approach to Late Iron Age and Roman Landscapes on the Northern Plateau, Spain, European Journal of Archaeology 16 (2), 289-313. DOI: https://doi.org/10.1179/1461957112Y.0000000027

GARCÍA SÁNCHEZ, J.; CISNEROS, M. (2014): Tisosa: Un establecimiento suburbano de Segisamo (Sasamón, Burgos), Archivo Español de Arqueología 87, 123-40. DOI: https://doi.org/10.3989/aespa.087.014.008

GARCÍA SÁNCHEZ, J.; COSTA-GARCÍA, J. M. (2019): El oppidum del Cerro de Castarreño, Olmillos de Sasamón. Historiografía y arqueología de un hábitat fortificado de la Segunda Edad del Hierro, Boletín de la Institución Fernán González 258, 9-45.
GARCIA SANCHEZ, J.; COSTA GARCÍA, J. M. (2020): Del oppidum indígena a la ciudad romana. Evolución del paisaje de Segisamo (Sasamón, Burgos, España) a través de la fotografía aérea. Datos de la prospección aérea de 2019, Lucentum XL, 131-148. DOI: https://doi.org/10.14198/LVCENTVM2020.39.07

GARCÍA SÁNCHEZ, J.; COSTA-GARCÍA, J. M.; FERNÁNDEZGÖTZ, M.; TEICHNER, F. (2020): Proyecto de prospección arqueológica. Warscapes: documentando la presencia militar romana en La Campiña (Sasamón, Burgos), Informe Final -Expte. 31/2017-BU. Mérida.

GIRÓN, L.; COSTA GARCÍA, J. M. (2009): La cerámica de imitación de engobe rojo pompeyano en los yacimientos militares romanos peninsulares. Un estado de la cuestión, Limes XX Congreso Internacional de Estudios sobre la Frontera Romana (A. Morillo, N. Hanel, M. Martín, eds.), León, 497-512.

GÓMEZ PANTOJA, J. L. (2000): Legio IIII Macedonica, Les Légions de Rome sous le Haut-Empire: actes du Congrès de Lyon (17-19 septembre 1998) (Y. Le Bohec, C. Wolff, eds.), Paris, 104-117.

PÉREZ GONZÁLEZ, C.; ILLARREGUI GÓMEZ, E. (2006): Producciones militares en el campamento de la "legio IIII Macedonica" en Herrera de Pisuerga, Arqueología militar romana en Hispania II : producción y abastecimiento en el ámbito militar (A. Morillo, ed.), León, 111-183.

GRAU MIRA, I. (2017): Archaeological surveys in areas with a high density of artefacts: Analysis and interpretation proposals, Quaternary International 435 (B), 71-80. DOI: https://doi.org/10.1016/j.quaint.2015.12.063

GURT, J. M.; RODÀ DE LLANZÁ, I. (2005): El Pont del Diable. El monumento romano dentro de la política territorial augustea, AEspA 78, 147-165. DOI: https://doi.org/10.3989/aespa.2005.v78.78

LAPUENTE, M. P.; PEREZ-ARANTEGUI, J.; AGUAROD OTAL, C.; ALCORTA, E. (1996): Caracterización de imitaciones provinciales micaceas de engobe rojo-pompeyano en el norte de la Península Ibérica, Actes du colloque de Périgueux 1995 (M. Di Filippo, O. Grubessi, B. Toro, eds.), Supplément à la Revue d'Archéometrie, 89-94.

LINARES, J.; HUERTAS, F.; CAPEL, J. (1983): La arcilla como material cerámico. Características y comportamiento, CPAUG 8, 479-490.

LÓPEZ NORIEGA, P. (1997): Organización territorial romana en el conventus cluniensis, Zephyrus 50, 217-24.

LÓPEZ NORIEGA, P. (1998): Proceso de urbanización en época romana: algunos ejemplos del conventus cluniensis, Los orígenes de la ciudad en el noroeste hispánico. Actas del Congreso Internacional I (A. Rodríguez Colmenero, ed.), Lugo, 643-52.

LOSTAL PROS, J. (2009): Los miliarios de la via romana de las Cinco Villas y del Pirineo aragonés, Item a Caesarea Augusta Beneharno: la carretera romana de Zaragoza al Bearn (I. Moreno, ed.), Ejea de los Caballeros, 191-237. 
¿LABRA FICTILIA? UNA PROPUESTA INTERPRETATIVA PARA TRES TERRACOTAS DECORADAS PROCEDENTES DE SEGISAMO (SASAMÓN, BuRGOS) Y Villabermudo (Palencia)

MARTINEZ CABALLERO, S.; SANTOS YANGUAS, J.; MUNICIO GONZÁLEZ, L. (Eds.) (2018): El urbanismo romano en el valle del Duero. Actas de la I Reunión de ciudades romanas del valle del Duero. Segovia, 20 y 21 de octubre de 2016, Segovia.

MAYORAL HERRERA, V.; URIARTE, A. (2011): 'Background noise' and landscape exploitation in the Late Iron Age Andalusian countryside, Hidden Landscapes of Mediterranean Europe. Cultural and methodological biases in preand protohistoric landscape studies (P. M. Van Leusen, G Pizziolo, L. Sarti, eds.), London, 265-270.

MENÉNDEZ BLANCO, A.; GARCÍA SÁNCHEZ, J.; COSTAGARCÍA, J. N.; FONTE, J.; GONZÁLEZ-ÁLVAREZ, D.; VICENTE GARCÍA, V. (2020): Following the Roman Army between the Southern Foothills of the Cantabrian Mountains and the Northern Plains of Castile and León (North of Spain): Archaeological Applications of Remote Sensing and Geospatial Tools, Geosciences 10 (12), 485.

DOI: https://doi.org/10.3390/geosciences10120485

MORILLO CERDÁN, Á. (1992): Cerámica romana de Herrera de Pisuerga (Palencia - España). Las lucernas, Santiago de Chile.

MORILLO CERDÁN, Á. (2000): La legio IIII Macedonica en la península Ibérica. El campamento de Herrera de Pisuerga (Palencia), Les Légions de Rome sous le Haut-Empire: actes du Congrès de Lyon (17-19 septembre 1998) (Y. Le Bohec, C. Wolff, eds.), Paris, 609-624.

MORILLO CERDÁN, Á.; PÉREZ GONZÁLEZ, C.; ILLARREGUI GÓMEZ, E. (2006): Herrera de Pisuerga (Palencia). Cronologías estratigráficas: cerámicas y monedas, Los campamentos romanos en Hispania (27 a.C.-192 d.C): el abastecimiento de moneda (M. P. García-Bellido, ed.), Madrid, 324-337.

MORILLO CERDÁN, Á.; SALIDO DOMÍNGUEZ, J. (2010): "Labrum" romano procedente de las termas del campamento de la "Legio VII gemina" en León, Zephyrus 65 167-178.

MORILLO CERDÁN, Á.; SALIDO DOMÍNGUEZ, J. (2011): Labra de época romana en Hispania, AEspA 84, 153-78. DOI: https://doi.org/10.3989/aespa.084.011.006

MORILLO CERDÁN, Á.; SALIDO DOMÍNGUEZ, J. (2013): Marcas militares sobre producciones latericias en Hispania. Nuevas consideraciones sobre su origen y difusión, Gerión 31, 287-329.

DOI: https://doi.org/10.5209/rev_GERI.2013.v31.43623
PALAO VICENTE, J. J. (2017): Milites in urbibus: La presencia de soldados en las ciudades hispanas durante el Alto Imperio, Memoriae civitatum: arqueología y epigrafía de la ciudad romana: estudios en homenaje a José Manuel Iglesias Gil (A. Ruiz, C. Cortés, eds.), Santander, 51-80.

PÉREZ GONZÁLEZ, C. (1983): Cajitas celtibéricas de la provincia de Palencia: aportaciones, síntesis y localización, Publicaciones de la Institución Tello Téllez de Meneses 48, 5-27.

PÉREZ GONZÁLEZ, C. (1993): Villabermudo: la villa romana más al norte de Palencia, Apuntes Históricos Herrera de Pisuerga 17, 5-23.

PÉREZ GONZÁLEZ, C. (2017): Nuevos datos para un mejor conocimiento histórico-arqueológico del asentamiento hispanorromano de Villabermudo de Ojeda (Palencia), Oppidum: cuadernos de investigación 13, 105-29.

PÉREZ GONZÁLEZ, C.; ILLARREGUI, E. (1990): Las llamadas cajitas de celtibéricas de época romana de Villabermudo, Actas del II Congreso de Historia de Palencia (M. V. Ca1leja, coord.), Palencia, 297-317.

PÉREZ GONZÁLEZ, C.; ILLARREGUI, E. (2006): Producciones militares en el campamento de la Legio IIII Macedonica en Herrera de Pisuerga, Arqueología militar romana en Hispania II: producción y abastecimiento en el ámbito militar (Á. Morillo, ed.), León, 111-134.

QUINN, P. (2013): Ceramic Petrography: The Interpretation of Archaeological Pottery \& Related Artefacts in Thin Section, London. DOI: https://doi.org/10.2307/j.ctv1jk0jf4

RAMOS SAINZ, M. L. (1996): Las terracotas arquitectónicas en la Hispania romana: la tarraconense, Tesis Doctoral, Universidad Autónoma de Madrid.

SACRISTÁN DE LAMA, J. D. (2011): El urbanismo vacceo, Complutum 22 (2), 185-222. DOI: https://doi.org/10.5209/rev_CMPL.2011.v22.n2.37731

SEVILLANO PEREA, L. A.; MAYORAL HERRERA, V.; SALAS TOVAR, E.; LICERAS, R.; HERAS MORA, F. J. (2013): Detectando prácticas agrarias antiguas en el territorio sur de Medellín. La expresión material de las actividades agrícolas protohistóricas del Suroeste peninsular, VI Encuentro de Arqueología del Suroeste Peninsular, Villafranca de los Barros (J. Jiménez Ávila, M. Bustamante-Álvarez, M. García Cabezas, coords.), Villafranca de los Barros, 1031-1064.

SOLANA SÁINZ, J. M. (2001): Los turmogos: territorio y modos de vida, Indígenas y romanos en el norte de la Península Ibérica (J. Santos Yanguas, ed.), Donostia, 95-118.

TEJA CASUSO, R. (1991): Turmogos, Las entidades étnicas de la Meseta Norte de Hispania en época romana (J. M. Solana, coord.), Valladolid, 193-210. 